\title{
DINÁMICA DE LA ESTRUCTURA ORGANIZACIONAL
}

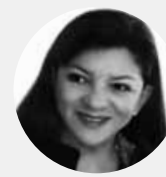

LAURA HUAMÁN PULGAR - VIDAL

- Profesora de Diseño Organizacional Área Académica de Administración Facultad de Negocios, UPC

\section{INTRODUCIÓN}

La estructura organizacional en la gestión de las organizaciones puede ser un tema de fácil entendimiento a nivel teórico. Sin embargo, las bondades de la teoría y sus conceptos llegan a ser limitantes cuando se trata de llevarlo a la práctica.

Si bien la estructura organizacional se elabora en la fase "organizar", del clásico proceso administrativo y luego de la "planeación", la relación que existe entre el hecho de planear y organizar es clave cuando se crea una estructura organizacional. Las teorías y los métodos afines son diversos, habiéndose planteado variados modelos y metodologías. Quien dirija y controle la ejecución de los planes tendrá que tener mucha claridad en el orden de su organización y para eso la definición y claridad de una estructura será uno de los más importantes medios para el éxito de la estrategia.

Es gracias a la docencia y al ejercicio de la consultoría de empresas que se evidenció la oportunidad de revisar el tema de la creación de estructuras organizacionales y presentar una propuesta metodológica al respecto.

\section{ANTECEDENTES}

El concepto de organización se ha definido en el campo de la administración desde varias perspectivas: social, económica o de sistemas. Pero su esencia consiste en concebirla como una entidad donde dos o más personas trabajan en conjunto para lograr objetivos compartidos. Esta organización humana donde se transforman recursos para ciertos fines opera como un sistema; no está aislada, vive inmersa en un sistema superior a ella donde hay otras organizaciones con las cuales interactúa; mantiene relaciones de intercambio; da y recibe influencias desarrollándose una dinámica conjunta.

Esta organización ha de definir cómo posicionarse y desenvolverse en ese medio que la rodea en función de los objetivos para los que se creó, vale decir, su propósito o misión; y, justamente, es en esta situación donde gracias al proceso de planeación, define una estrategia que habrá de ejecutarse en un plazo de tiempo

1. Los autores son Franklin Gerald Ríos y Laura Huamán Pulgar Vidal, -autora del artículo- profesores de la Universidad Peruana de Ciencias Aplicadas. Actualmente ya se han publicado más de dos mil ejemplares en dos ediciones por la Editorial UPC y hay una versión internacional para Latinoamérica en alianza con las Ediciones de la U, impreso en Colombia en abril del presente año. determinado y que requerirá, además de muchos tipos de recursos, de una estructura organizacional.

La estrategia puede estar muy bien concebida y representar para las personas de la organización una forma de orientar el trabajo, de utilizar los recursos, la tecnología y las habilidades humanas, retos grupales e individuales, éxito y rentabilidad.... No obstante, llegará un momento en el cual el soporte y apoyo de la estructura será un elemento clave y no menos importante que el equipo directivo con sus competencias organizacionales necesitarán para abordar las demandas del entorno. Estamos hablando de la creación de una organización capaz de ejecutar exitosamente una estrategia.

Hemos observado en la práctica que aunque existe el propósito, la estrategia y el plan en las organizaciones, la estructura no siempre apoya a la ejecución de la estrategia; no está alineada o en el peor de los casos ha tardado en tener la forma y dinámica que ésta requiere. Este hecho se ha manifestado en diversas organizaciones sin mayor diferencia en cuanto a tamaño y actividad, al igual que hay en común ciertos requerimientos similares y adopción de ciertos cambios organizacionales que se reflejan en la estructura. Iremos paso a paso, en lo que resta del presente documento.

\section{MODELO PROPUESTO: METODOLOGÍA PARA EL DISEÑO DE LA ESTRUCTURA ORGANIZACIONAL}

La metodología que se presenta a continuación y que es para el diseño de la estructura organizacional vinculada a la estrategia empresarial, es producto de una investigación de largos años que se validó oportunamente y que forma parte del libro Metodologías para implantar la estrategia: diseño organizacional de la empresa ${ }^{1}$, publicado en 2011 y que es, actualmente, uno de los principales textos para enseñanza del tema, materia del presente artículo

Tal como su nombre lo expresa surgió como tema de investigación y estudio a partir desde dos hechos observados y de distinto origen. El primero, las necesidades de las empresas en las que se trabajaba justamente el tema de organización que requería de enfoques prácticos; $\mathrm{y}$, originado como segundo, en el mundo de la teoría administrativa y de la organización; la necesidad de contar con una metodología que pudiera concretar, paso a paso, cómo hacerlo, incorporando lo que ya existía de valiosos teóricos y otros métodos que se fueron desarrollando unidos al desarrollo de la informática. Esta metodología se sustenta en una herramienta de Excel al que llamaremos "Macroestructura", que como aplicativo permite su desarrollo y además procesar la información. 
Figura 1. Metodología para el diseño de la estructura organizacional

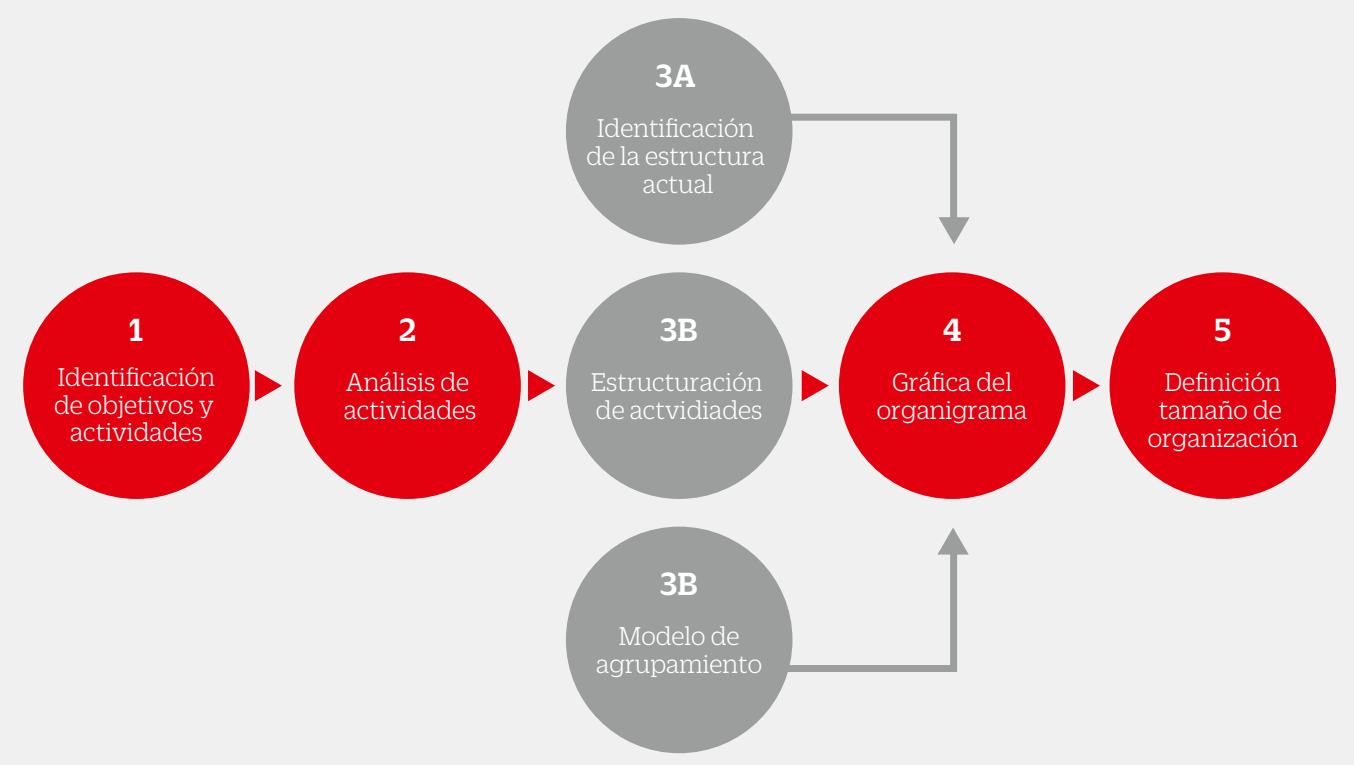

Fuente: Adaptado de Metodologías para implantar la estrategia: diseño organizacional de la empresa (Segunda ed.). Lima: Universidad Peruana de Ciencias Aplicadas (UPC), por L. Huamán y F. Ríos, 2011.

\section{Identificación de Objetivos y Actividades}

Existe una relación entre la planeación y la organización, de allí que organizar, definido como el proceso del diseño de la estructura, puestos de trabajo y los procesos, tiene como insumos dos componentes de la planeación que son los objetivos y las actividades, lo cual se observa en la figura 2. Esta parte de la metodología tiene como objetivo sólo el diseño de la estructura organizacional, siendo otra la metodología que se emplea para el diseño de los puestos de trabajo y otra para los procesos.

Estos insumos, objetivos y actividades, son especificados en detalle para poder poner en marcha la estrategia. Así, pueden describirse múltiples actividades a realizar de manera independiente y a la vez relacionarlas al logro de los objetivos.

Figura 2. Estrategia y organización: alineación de la estrategia

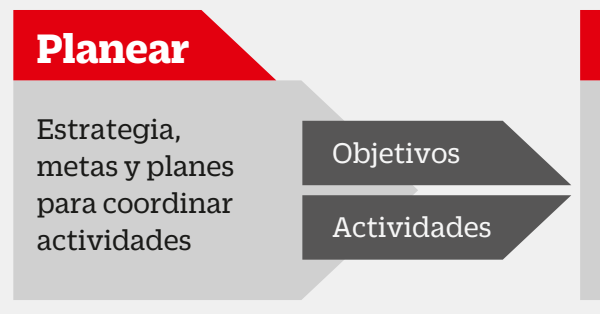

\section{Organizar}

Estructura organizacional

Puestos de trabajo

Procesos

Fuente: Adaptado de "Metodologías para implantar la estrategia: diseño organizacional de la empresa (Segunda ed.). Lima: Universidad Peruana de Ciencias Aplicadas (UPC), por L. Huamán y F. Ríos, 2011. 
TABLA 1: Identificación y análisis de actividades.

Organización: Club Paraíso

\section{N Objetivo}

1 Crecimiento

2 Mejora del Servicio

3 Mejora del Servicio

4 Mejora del Servicio

5 Mejora del Servicio

6 Prestigio Internacional

7 Mejora del Servicio

8 Mejora del Servicio

9 Mejora de Servicio

10 Prestigio Internacional

11 Prestigio Internacional

12 Mejora del Servicio

13 Prestigio Internacional

14 Mejora de Servicio

15 Mejora del Servicio

16 Prestigio Internacional

17 Mejora de Servicio

18 Prestigio Internacional

\section{Actividad}

Gestionar financiamiento para inversiones en infraestructura de las diversas sedes.

Implementar sistemas informaticos para interconexion entre las sedes.

Capacitar al personal en nuevas tecnologias

Crear competencias deportivas internas para los socios y sus familiares.

Innovar los procesos de servicio con tecnologia de punta para las operaciones del club.

Promocionar y consolidar la imagen del club en eventos nacionales como internacionales.

Impulsar los eventos sociales y empresariales para que tengan alto impacto.

Implementar un portal Web para promocionar los eventos del club y mantener informados a los socios.

Implementar sistema de mantenimiento preventivo de las instalaciones deportivas y sociales del club.

Evaluar y consolidar los torneos y eventos sociales con ambito internacional para ser sede o participar.

Buscar y consolidar alianzas estrategicas cono clubes del exterior.

Mejorar el servicio y atencion al cliente con estandares internacionales.

Conseguir financiamaiento para competencias y torneos deportivos internacionales.

Pagar estudios de especializacion para empleados competentes.

Considerar a los empleados en la reparticion de las utilidades de la empresa.

Mejorar los eventos sociales y empresariales para que sean de ambito internacional.

Incentivar a los slcios en la pracitca de los deportes.

Captacion de socios de clase A, con alta imagen publica nacional e internacional.

Fuente: Adaptado de "Metodologías para implantar la estrategia: diseño organizacional de la empresa (Segunda ed.). Lima: Universidad Peruana de Ciencias Aplicadas (UPC), por L. Huamán y F. Ríos, 2011.

2. Tomado de Ríos y Huamán (2011), capítulo3. 
El ejemplo presentado explica de manera sencilla cómo empezar a hacer la estructura de la organización, especificando los objetivos que se desean lograr y las actividades para su desarrollo, lo que significa que un mismo objetivo puede requerir para su realización más de una actividad. Puede observarse que las actividades describen lo que hay que hacer de manera concreta, específica, estable y se expresan de forma directa, simple y breve. Esta parte es el nexo entre la planeación y el proceso de diseñar la organización.

\section{Análisis de las Actividades}

Esta segunda etapa evalúa a cada una de las actividades en términos del aporte de cada una de ellas a los resultados y del desempeño admisible de cada una. No todas las actividades aportan a los resultados de forma directa y son evidentemente visibles; por esta razón, algunas aportan directamente, otras de manera complementaria y otras pueden ser accesorias pero de ninguna manera prescindibles en el conjunto de los resultados y su sinergia. Tenemos, por ejemplo, las ventas, la implementación de un sistema informático que toma su tiempo y la preparación de la infraestructura física. En cuanto a desempeño, pueden considerarse aquellas en donde no es posible tener falla o cometer error alguno pues esto costaría la no realización de la estrategia; otras actividades pueden estar en prueba, por ejemplo implementar un nuevo sistema informático y otras que pueden estar en experimentación o cuya falla o deficiencia no implica el sacrificio en el logro de la estrategia.

Esta evaluación de aporte y desempeño otorga un puntaje a la actividad, pudiendo en conjunto tener tres tipos de actividades clasificadas por grados de sensibilidad con relación a la ejecución de la estrategia. La evaluación de las mismas requiere de un claro conocimiento de la organización y de buenas habilidades de análisis.

El análisis de sensibilidad se complementa con el grado de relación entre las actividades que pude ser directa (relación A), vinculada en el proceso (relación B) o que no exista relación (relación C). En el aplicativo se observa este resultado - ver figura 3.

Figura 3. Aplicativo macroestructura: análisis de actividades

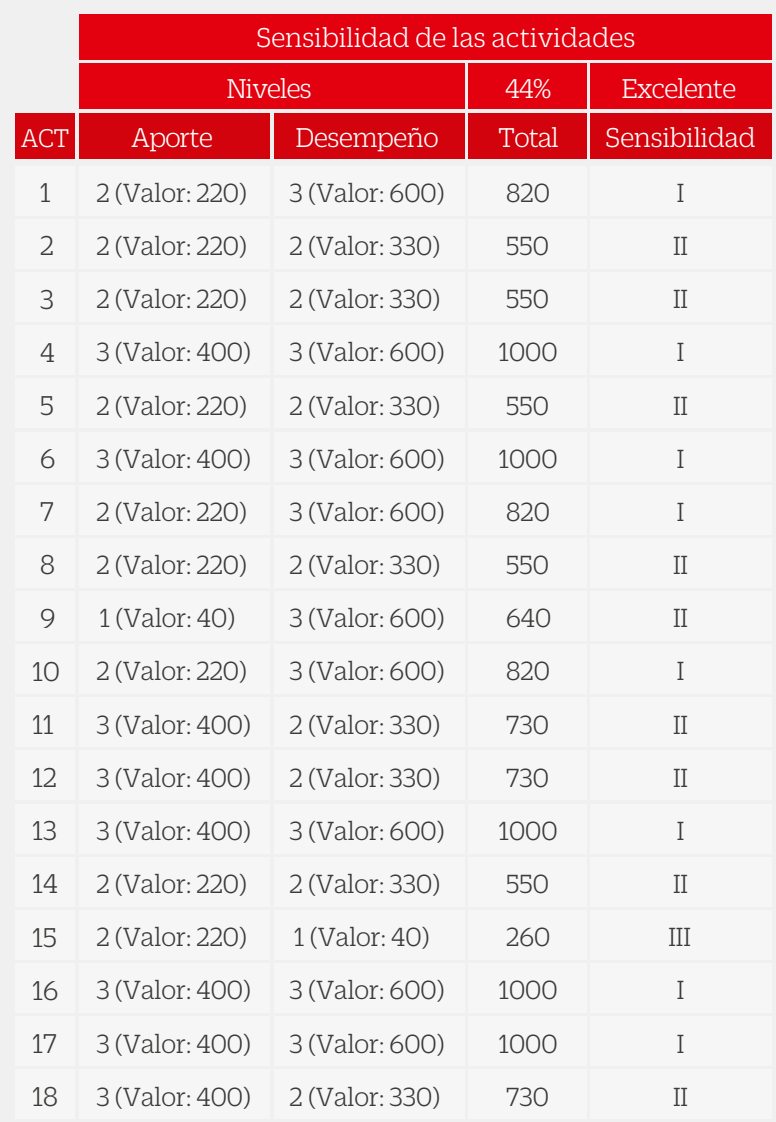

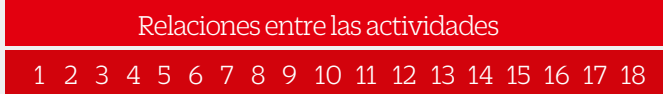

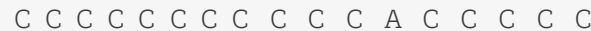

C C C C C B C C C C C C C C C C

C C C C C C C C C C A B C C C

C C C C C A C C C C C C A C

C C C A C C C C C C C C C C

C C C C A C C C C C C A

C C C C C C C C C C C A C C

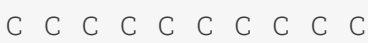

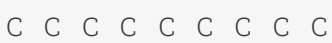

C C C C C C C B C

B C C C C C C C B

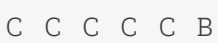

C C C C C

A C C C

C C C

C C

C

Fuente: Adaptado de "Metodologías para implantar la estrategia: diseño organizacional de la empresa (Segunda ed.). Lima: Universidad Peruana de Ciencias Aplicadas (UPC), por L. Huamán y F. Ríos, 2011. 
Observando la figura surge una interrogante con relación a la calificación de "Excelente" y que significa en la práctica que la proporción de actividades según la sensibilidad que tienen es apropiada dentro de un rango en el cual se tienen actividades de gran significado para la estrategia y que hay otras de naturaleza complementaria y operativa que igualmente son necesarias de realizar. Sin embargo ¿cómo sería si sólo hubiera actividades de un solo tipo? Por ejemplo, todas de sensibilidad I. Ello significaría que todo es crítico y para ahora mismo, no dejando tiempo al proceso de implantación de la estrategia que toma su tiempo y maneja prioridades y criterios de urgencia e importancia.

\section{Procesamiento de la Información}

En esta parte (3A), se toma la estructura inicial de la organización, que en muchos casos está graficada según el criterio de la empresa y refleja lo que se realiza en la actualidad, que, inclusive, al requerirla a veces no existe. La estructura inicial del Club Paraíso, para continuar con el ejemplo, se muestra en la figura 4:

Figura 4. Estructura del Club Paraíso - tipo funcional

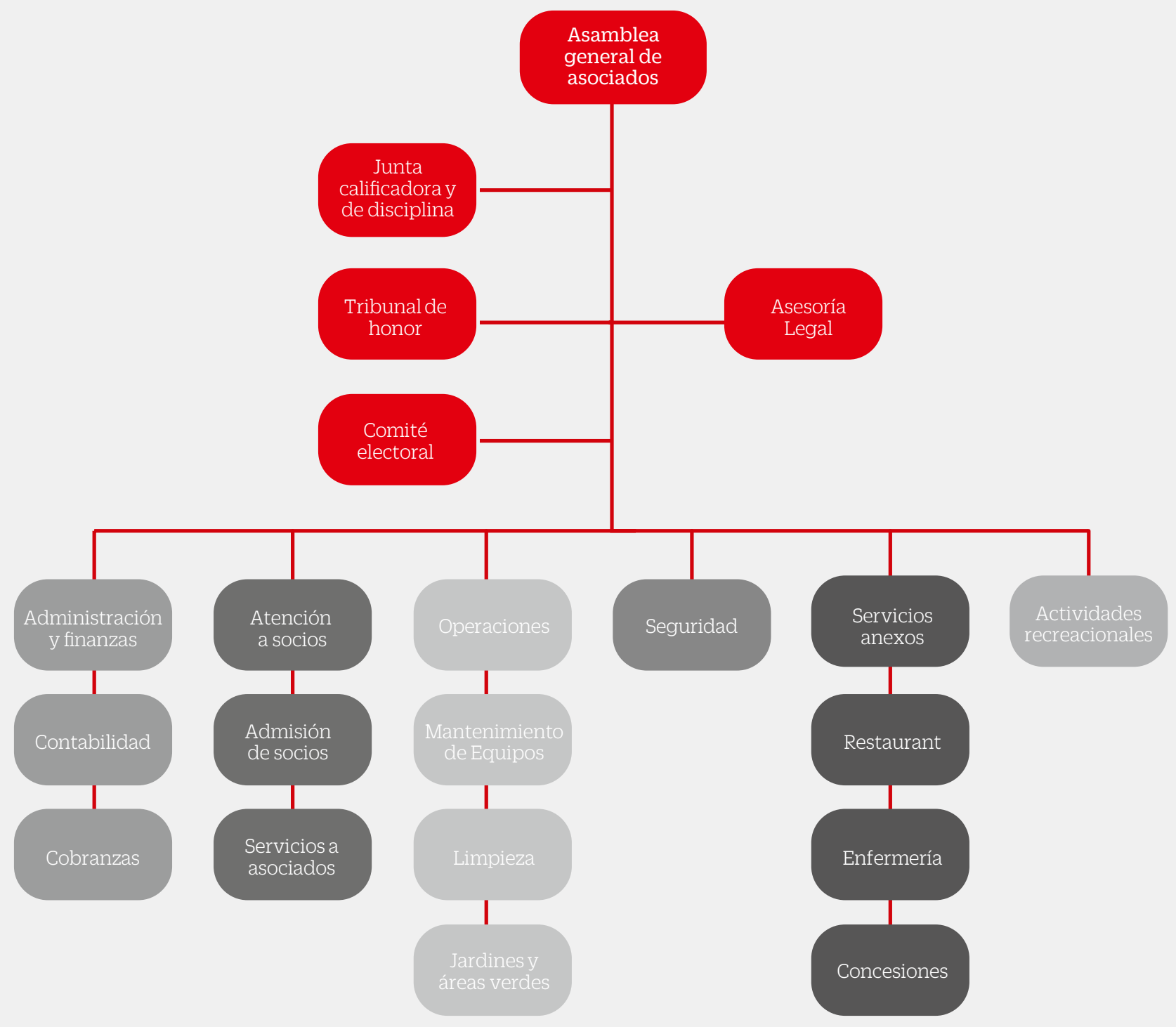

Fuente: Adaptado de "Metodologías para implantar la estrategia: diseño organizacional de la empresa (Segunda ed.). Lima: Universidad Peruana de Ciencias Aplicadas (UPC), por L. Huamán y F. Ríos, 2011. 
La estructuración de las actividades (3B), consiste en su procesamiento manual, agrupándolas según su sensibilidad y relación, dando lugar a la formación de unidades organizativas que pueden ser nuevas o con nombre igual a otras ya existentes que se agregarán a las de estructura inicial (3A). En esta etapa el analista debe poner el nombre apropiado a cada unidad organizativa de tal forma que refleje una función para la ejecución de la estrategia. En la figura 5 y la figura $\mathbf{6}$ se presentan la formación de las unidades organizativas, producto del procesamiento manual y la estructura resultante:

Figura 5. Unidades organizativas formadas por el procesamiento de la información (tres líneas representan la relación tipo A y una línea representa la relación tipo B)
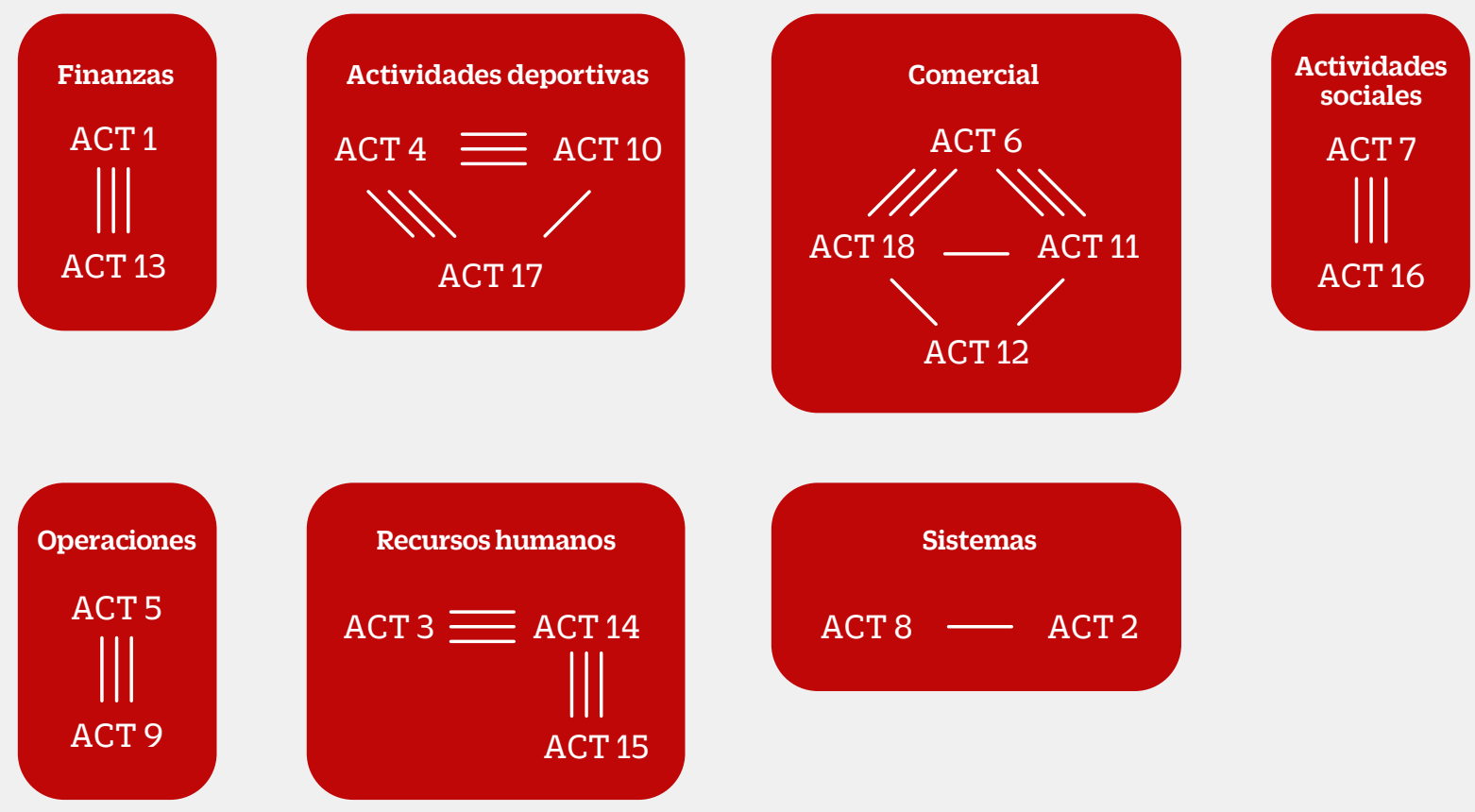

Fuente: Adaptado de "Metodologías para implantar la estrategia: diseño organizacional de la empresa (Segunda ed.). Lima: Universidad Peruana de Ciencias Aplicadas (UPC), por L. Huamán y F. Ríos, 2011.

Figura 6. Estructura Resultante: se agrupa en un organigrama las unidades organizativas de la figura 5

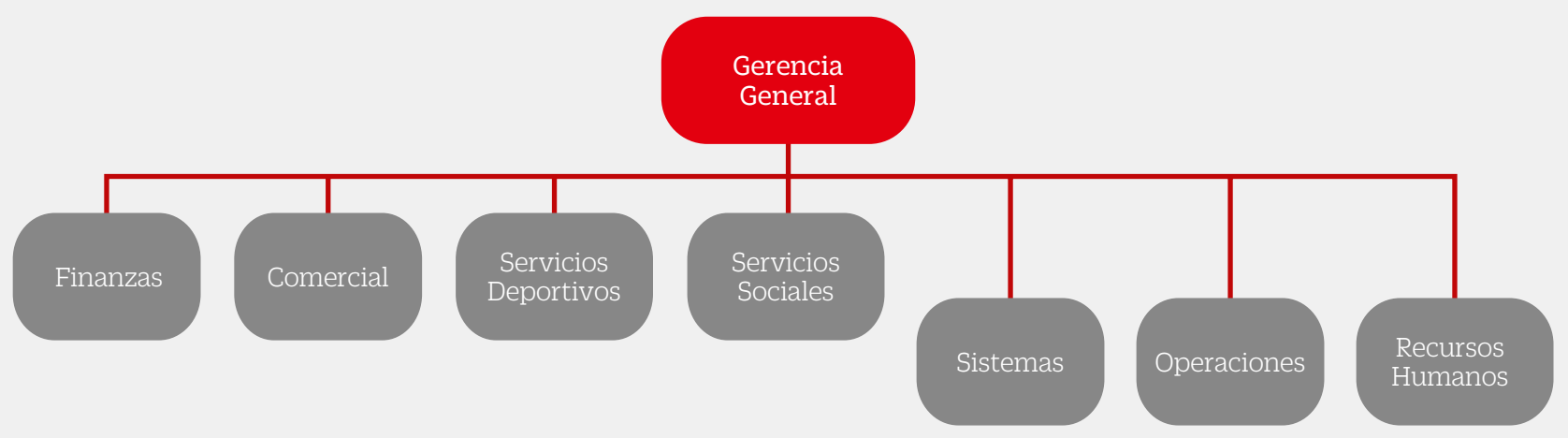

Fuente: Adaptado de “Metodologías para implantar la estrategia: diseño organizacional de la empresa (Segunda ed.). Lima: Universidad Peruana de Ciencias Aplicadas (UPC), por L. Huamán y F. Ríos, 2011. 
Finalmente, el paso (3C), consiste en identificar el modelo de agrupamiento original. Refleja cómo se relacionan y vinculan las actividades preexistentes con las nuevas a través de las decisiones de diseño, donde se realizan varios ajustes consistentes con un patrón de ordenamiento que luego permite graficar el organigrama. Las decisiones de diseño consideran y destacan la formación de unidades organizativas - ajuste en la estructuración la conservación o no de unidades organizativas en la estructura final - decisiones de comparación entre las estructuras - y decisiones para elegir un modelo de agrupamiento - que puede consistir en una nueva forma de agrupamiento para el organigrama.

En el caso del ejemplo, al organigrama funcional original del Club Paraíso (figura 4) se le incorpora y adiciona el organigrama resultante (figura 6), que representa y refleja las actividades que hay que realizar para el logro de la nueva estrategia mediante las decisiones de diseño ya mencionadas. En este caso es importante comparar la estructura inicial con la resultante para determinar qué unidades organizativas están ausentes en la nueva estructuración para adicionarlas; cuales actividades son adicionales a unidades ya existentes para mostrar sus cambios; cuales cambian de posición; cuales se podrían tercerizar; o cuales cerrar, porque no contribuyen a los objetivos empresariales.

\section{Gráfica del organigrama final}

Se emplean las técnicas convencionales para la gráfica de organigramas y se utilizan los nombres según la teoría de la organización clásica. Aquí pueden utilizarse la estructura funcional, matricial, divisional, por productos, por procesos, geográfica, por clientes, multidivisional o híbrida (que combina dos o más formas de agrupación), según lo que se ha graficado. El concepto de Henry Mintzberg sobre la división fundamental del trabajo que se representa en su modelo de las partes básicas de la organización es utilizado para ordenar las diferentes unidades organizativas según el rol que tienen en la organización como conjunto-- véase la figura 7.

\section{Figura 7. División fundamental del trabajo, partes básicas de una organización}

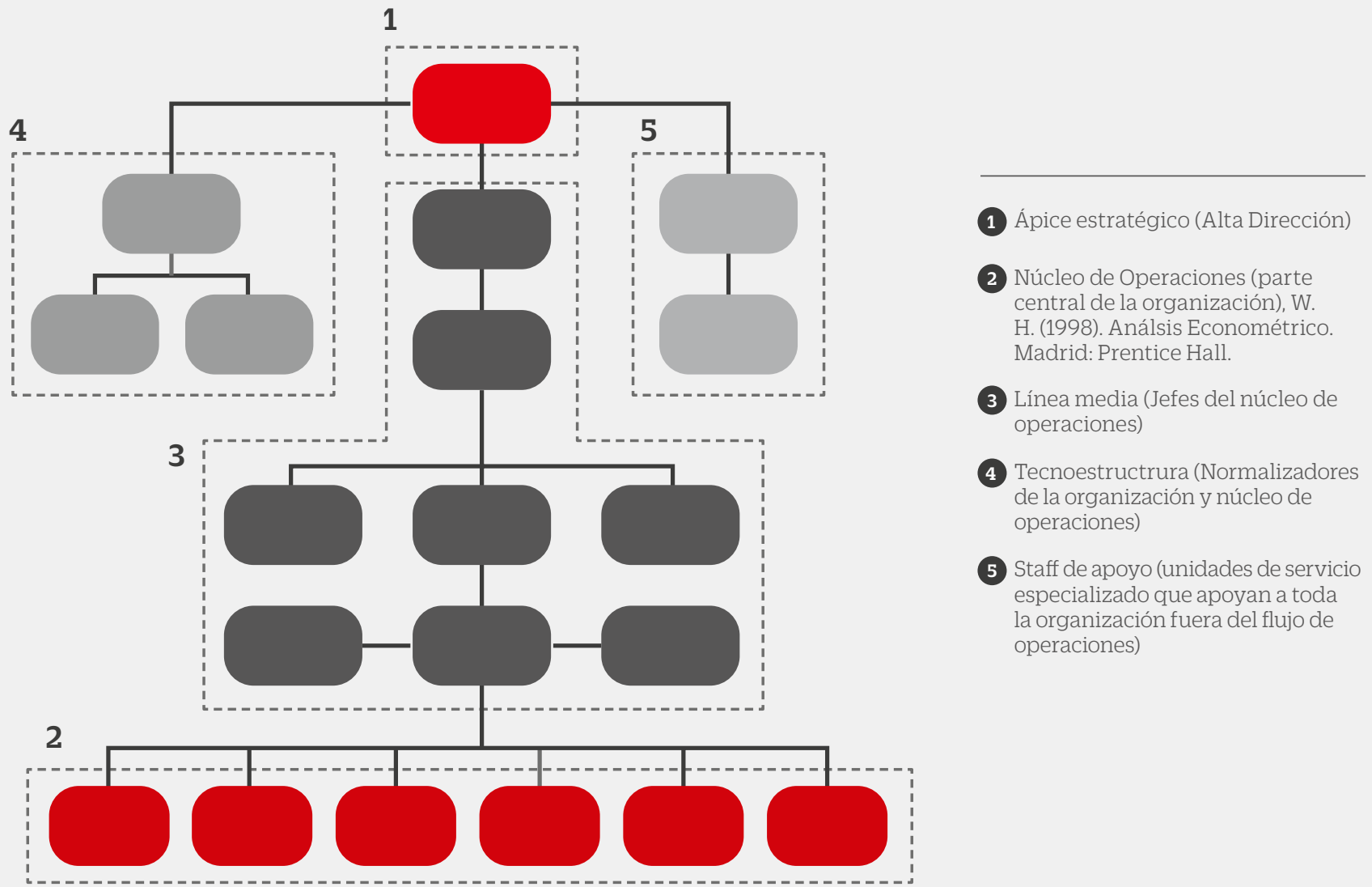

Fuente: Adaptado de "Metodologías para implantar la estrategia: diseño organizacional de la empresa (Segunda ed.). Lima: Universidad Peruana de Ciencias Aplicadas (UPC), por L. Huamán y F. Ríos, 2011. 
Figura 8. Estructura final del Club Paraíso

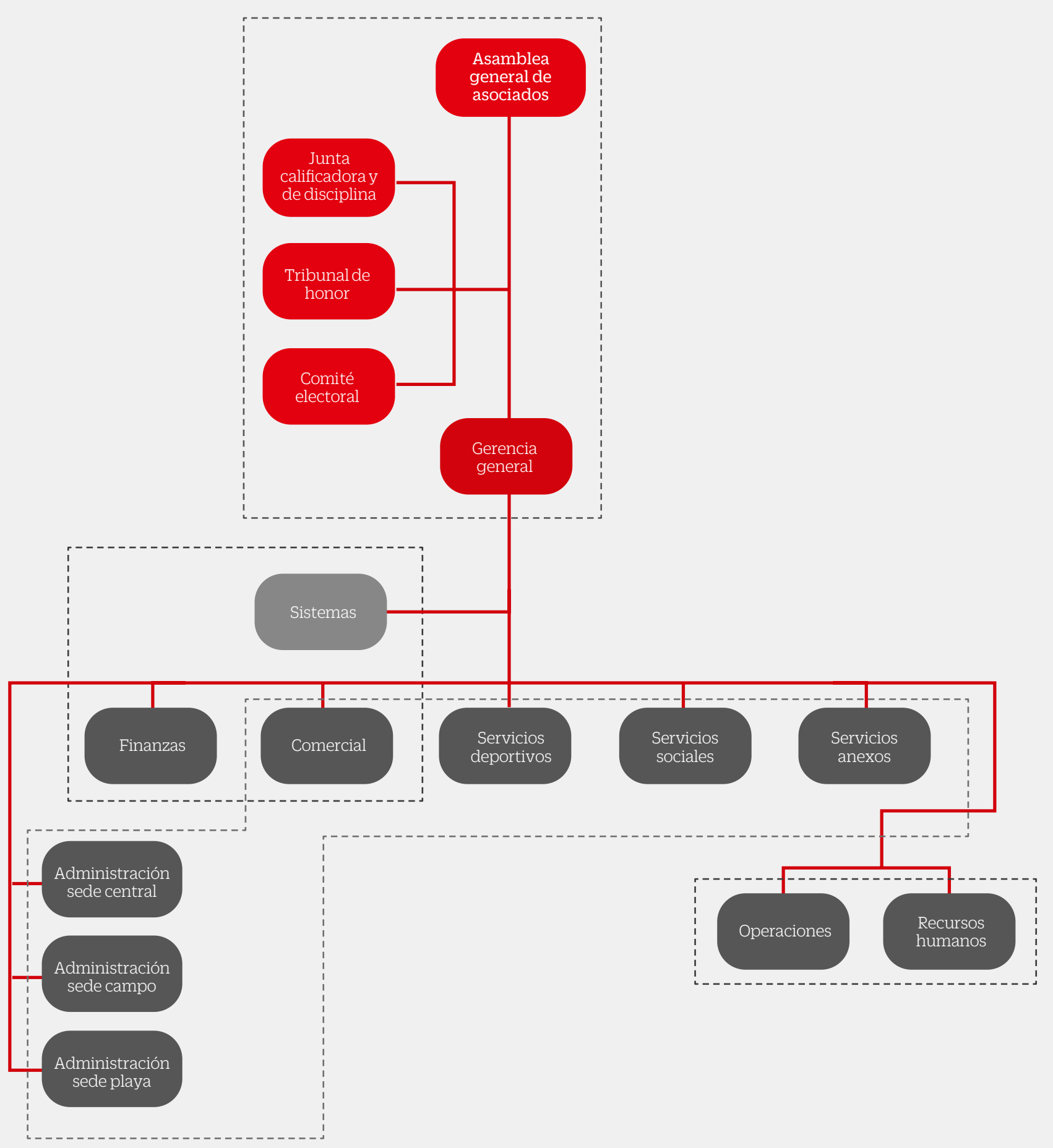

Fuente: Adaptado de "Metodologías para implantar la estrategia: diseño organizacional de la empresa (Segunda ed.). Lima: Universidad Peruana de Ciencias Aplicadas (UPC), por L. Huamán y F. Ríos, 2011. 
Se muestra el modelo de agrupamiento matricial. En naranja Unidades de Ápice Estratégico, en azul la Tecnoestructura, en verde el Staff de Apoyo y en celeste el Núcleo de Operaciones donde se observa que según la nueva estrategia se implementarán tres sedes que ofrecerán los servicios correspondientes a un Club.

\section{Tamaño de la organización}

Se define en función de los requerimientos de la estrategia, es decir, que las actividades de una unidad organizativa pueden ser realizadas por una sola persona y en este caso es un puesto de trabajo o en su defecto, deben ser realizadas por un equipo de personas. En esta etapa se aplica otra metodología que es la del diseño del puesto de trabajo.

\section{Observaciones respecto a la metodología}

Se ha presentado el desarrollo sobre cómo hacer una estructura, que se origina y tiene como punto de partida a los objetivos y actividades de la estrategia proveniente de la planeación. La estructura representa lo que hay que hacer a través de diversas unidades organizativas debidamente ordenadas y agrupadas y está expuesta a elementos modeladores como son la tecnología, los recursos, las capacidades organizacionales, la dirección de estas actividades y al provenir de la planeación; puede considerarse alguna variante de la estructura como parte del proceso de cambio que implica la implantación de la estrategia. Esto implica que graficar una estructura de transición también es posible.

La propuesta metodológica es una parte del diseño organizacional. Se ha hecho breve referencia al diseño de los puestos pero queda en suspenso el diseño de los procesos que tiene como objetivo hacer funcionar la organización, que implica una propuesta dinámica y no estática de la estructura organizacional.

\section{HALLAZGOS RECIENTES EN LA APLICACIÓN DEL DISEÑO DE ORGANIZACIONES}

Comentamos algunos hechos reales encontrados en el desarrollo de estudios de diseño de estructuras organizacionales, que presentamos a continuación:

\section{Sobre la información para el estudio y la estructura inicial y final}

(1) De un total de 20 empresas estudiadas ${ }^{3}$ en los últimos dos años, el 70\% tenía un organigrama y el $30 \%$ restante graficó uno al momento de solicitarlo según interpretaban cómo estaban organizados. Estudiarlos y analizarlos fue una grata experiencia al observar las tantas formas de estructuras que reflejaban la organización familiar, la simplicidad, la multifuncionalidad y de alguna forma su estrategia.

3. Pequeñas empresas $80 \%$, de giros diversos como artesanía, servicios, agricultura, hoteles, restaurantes, peluquerías, agencias de aduana, empresas constructoras, agroindustria, colegios, centros de esparcimiento y recreo, haciendas, un cementerio, laboratorios, importadoras y exportadoras, fábricas de tintes, comercios, etc. En el $20 \%$ restante se estudiaron empresas de telefonía, industria ladrillera de refrescos, etc. Ver Ríos y Huamán (2011).
(2) Aplicando la metodología, se requirió información sobre el planeamiento estratégico y todas tenían un plan en mente (100\%). Sin embargo, al momento de aclarar los objetivos y definir las actividades, se tuvo que dar soporte a los empresarios para desarrollarlas ya que se solicitaba un mínimo de 30 actividades. Podría decirse que la organización de las actividades o la creación de unidades organizativas nuevas según nuevos objetivos, surgían según la necesidad mas no se anticipaba, lo que sustenta un estudio ya realizado sobre el tiempo que toma configurar la estructura para la estrategia.

3 En la parte de estructuración de las actividades, la identificación de la estructura actual y elaborar el modelo de agrupamiento tuvo que hacerse modificaciones a las estructuras originales en un 100\%.

4 De manera anecdótica, en las empresas familiares (varias de las estudiadas), la estructura final era para los trabajadores, pero no para la familia que se adicionaba a la estructura final ya establecida y en posiciones muy peculiares casi cuestionando el método empleado, así no podía prescindirse de ciertos familiares clave y de presencia simbólica.

\section{Sobre la creación de unidades organizativas en} el diseño organizacional

(1) Unidades más empleadas y que se crean o reformulan y clarifican para ejecutar la estrategia: operaciones (producción), comercial y marketing.

(2) Unidades que se desvinculan del trabajo de la gerencia porque se requiere atención a las personas: recursos humanos o gestión de personas, capacitación.

3 Unidades que se observan como necesarias además de la contabilidad: la tesorería, finanzas y su integración con la función contable.

4 Unidades de soporte y sistematización de las operaciones: sistemas para contabilidad, finanzas, control y comercio electrónico.

5 Hallazgo interesante es que la mayoría de empresas considera que las unidades comerciales y de marketing, deben tener soporte de sistemas para fortalecer el trabajo de comunicación con los clientes, en algunos casos, se ha diseñado el híbrido comercial -sistemas.

(6) En algunos casos, la unidad de proyectos especiales se ha adjudicado a la gerencia general y no como unidad independiente dado especialmente el tamaño de las organizaciones.

7 Finalmente el uso de la tercerización también ha sido una propuesta a la que se ha llegado con la finalidad de concentrar en las empresas aquello que es su competencia central.

PERSPECTIVA DEL DISEÑO ORGANIZACIONAL EN EL MARCO DE LA DIRECCIÓN ESTRATÉGICA

La estructura de una organización, el diseño de los puestos y el desarrollo de los procesos conforman el diseño organizacional según hemos planteado. 
Sin embargo, esto no es todo lo necesario, porque hay muchas formas de organización modernas donde el soporte de las tecnologías de la información les proporciona la fluidez y modernidad que requieren los procesos y las comunicaciones como medio de coordinación. De todos modos, la estructura será siempre un medio valioso de soporte para la misma estrategia.

A continuación se presenta y para concluir, dónde podría ubicarse a la estructura en una configuración más compleja pero que es necesario considerar con otras dimensiones para comprender como funcionan las organizaciones. Se trata de la compleja estructura Hipertexto de Nonaka Takeuchi (1995) cuya finalidad es la creación del conocimiento y el aprendizaje organizacional - ver figura 9. Se caracteriza por la presencia de tres niveles organizacionales en forma de capas superpuestas e interrelacionadas lógicamente que comparten en simultáneo información, textos y gráficos de fuentes internas o externas, en un proceso dinámico de creación de conocimiento a través de redes de información. Las capas son: capa "Base de Conocimiento" con una base subyacente - y - a nuestra interpretación equivale a una capa adicional donde están la visión corporativa, la cultura organizacional, las bases de datos y el sistema tecnológico;

\section{Figura 9. La estructura hipertexto de Nonaka Takeuchi}

la capa "Sistema de Negocios", que equivale a la estructura organizacional y la capa "Equipo de Proyectos", que equivale a la organización en funcionamiento a través de los equipos de trabajo.

Esta forma de organización aprovecha la flexibilidad de las formas orgánicas (Equipos de Proyectos), la estabilidad de las formas mecánicas (Sistema de Negocios), la capacidad de las organizaciones en red y capitalizan el conocimiento que adquieren a través de una visión corporativa (Base de Conocimiento).

En el modelo, la capa "Sistema de Negocios" es aquella donde retornan los miembros de los equipos de proyectos - procesos - luego de haber trabajado en la base del conocimiento; retoman a sus actividades y funciones cotidianas hasta participar nuevamente en un proyecto nuevo o para hacer su trabajo cotidiano. Esta capa constituye la organización formal y tiene características burocráticas y a diferencia de las demás, es la que determina los objetivos y pautas para el proceso de generación de conocimiento integrado y donde se representa la división del trabajo que conduce igualmente a la ejecución de la estrategia. Esta capa, que es la estructura organizacionalobsérvese en la figura 9, su ubicación al centro y conectada con las otras dos - se vincula con las demás sustentadas por la visión empresarial y la cultura organizacional, la capa del conocimiento creado y acumulado, y finalmente los equipos de proyecto que hacen funcionar a la organización.

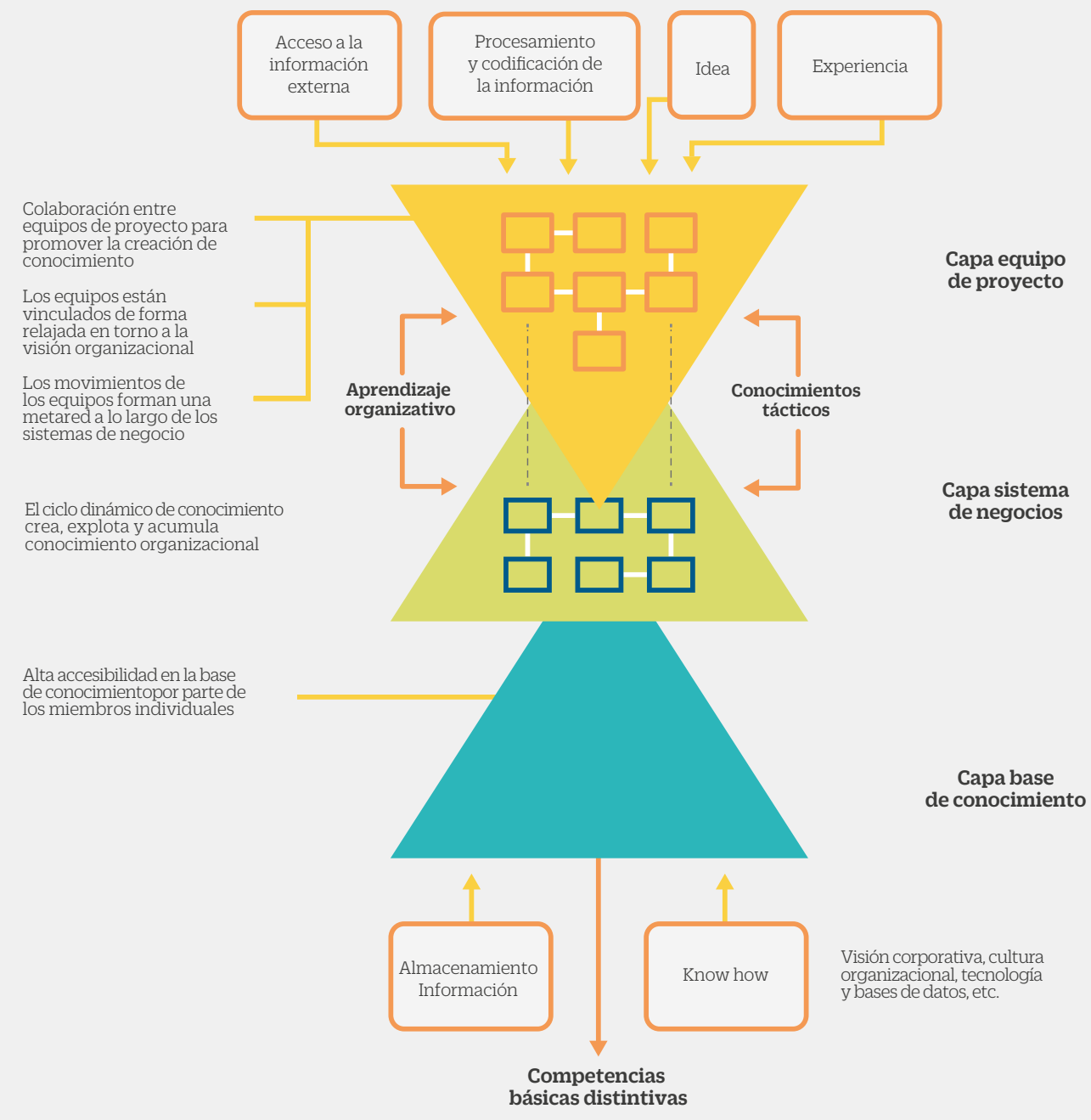

Adaptado de "La organización creadora de conocimiento. Cómo las compañías japonesas crean la dinámica de la innovación (Primera ed.). (M. H. Kocka, Trad.) México: Oxford University Press, por I. Nonaka y H. Takeuchi, 1995. 
Esta presentación gráfica aunque orientada a la gestión del conocimiento según sus autores, tiene a la estructura en el centro y es una de las mejores aproximaciones para comprender la importancia de la misma desde una perspectiva de dirección estratégica. Consideramos que esta estructura representa realmente y según nuestra interpretación, cómo funcionan las organizaciones en torno al diseño organizacional. Siendo así ¿cómo no enriquecer la teoría y aplicarla con nuevas y más renovadas configuraciones?

\section{CONCLUSIONES}

El diseño organizacional considera la estructura organizacional, el diseño de los puestos de trabajo y el diseño de los procesos de trabajo. La estructura de las organizaciones es un medio para lograr la estrategia empresarial y se requiere de un buen diseño organizacional como soporte para su ejecución. La elaboración de una estructura organizacional se fundamenta en los objetivos y actividades provenientes del proceso de planeación.

Las estructuras organizacionales originales estudiadas no siempre reflejaban la estrategia empresarial, ni sus actividades estaban alineadas con la misma.

Es posible aplicar una metodología para diseñar una estructura organizacional que permita realmente ejecutar una estrategia, incorporando información importante de la planeación.

Las actividades que hay que desarrollar para el logro de objetivos que representan una estrategia, pueden ser de diferente naturaleza y diversas; no todas aportan de forma similar a los resultados ni tampoco tienen similar nivel de desempeño. Las actividades pueden clasificarse, relacionarse y agruparse de manera consistente generando nuevas unidades organizativas de trabajo. La estructura organizacional puede adoptar varias formas en su modelo de agrupamiento a través de las decisiones de diseño. La división fundamental del trabajo es un esquema que permite ordenar las unidades organizativas según el rol que cumplen en la organización como conjunto.

En los estudios realizados, las empresas de diverso giro y tamaño, no tienen claras todas las actividades a desarrollar para el logro de sus objetivos de planeación y sus estrategias, y; sus estructuras organizacionales reflejan su propio concepto de organización y normalmente es necesario hacerles alguna modificación.

Lo más interesante de las investigaciones realizadas es la creación de unidades organizativas híbridas donde se vinculan la función de sistemas con la función comercial, dando lugar al comercio electrónico; la función que más se independiza de la gerencia es la gestión de personas.

Las organizaciones funcionan más allá de lo que refleja la sola estructura organizacional, son dinámicas y esto puede graficarse por ejemplo con la configuración de la estructura hipertexto que muestra esquemáticamente como funciona una organización.

\section{REFERENCIAS BIBLIOGRÁFICAS}

Andersen, J. A. (2002). "Organizational Design: Two lessons to learn before reorganizing". International Journal of Organization Theory and Behavior, V(3 \& 4), $343-358$

Baligh, H. H., \& Burton, R. M. \& Obel, B. (Diciembre de 1996). "Organizational Consultant: Creating a useable theory for organizational design". Management Science, XVII(12), 1648-1662.

Bennett, J. W., Pernsteiner, T. E., Kocourek, P. F. \& Hedlund, S. B. (Marzo y Abril de 2001). "Organización frente a la Estrategia: Resolver la paradoja de la Alineación". Harvard Deusto Business Review, (101), 18-27.

Burton, R. M., Desanctis, G. \& Obel, B. (2006). Organizational Design. U.S.A.: Cambridge University Press

Daft, R. L. (1998). Teoría y Diseño Organizacional (Sexta. ed.).U.S.A.: Internacional Thomson Editores

De Cossio Vivanco, J. L. (1985). “Diseño y Dinámica Organizacional”. Lima: Santa Matilde.

De La Fuente, J. M., García-Tenorio Ronda, J., Guerras Martín, L. A., \& Gomez Barahona, H. J. (2000). Diseño Organizativo de la Empresa (Segunda ed.). Madrid: Editorial Civitas.

Galán Zazo, J. I. (2006). Diseño Organizativo. España: Thomson.

Huamán, L., \& Ríos Ramos, F. (2011). Metodologías para implantar la estrategia: diseño organizacional de la empresa (Segunda ed.). Lima: Universidad Peruana de Ciencias Aplicadas (UPC).

Hodge, B. J., Anthony, W. P. \& Gales, L. M. (1999). Teoría de la Organización (Quinta ed.). Madrid: Prentice Hall.

Mintzberg, H.(1994). Diseño de Organizaciones Eficientes. (Cuarta ed.). Buenos Aires: Editorial "El ateneo".

Mintzberg, H. (1991). Mintzberg y la Dirección. Madrid: Ediciones Díaz de Santos S.A.

Nonaka, I. y Takeuchi, H. (1995). La organización creadora de conocimiento. Cómo las compañías japonesas crean la dinámica de la innovación (Primera ed.). (M. H. Kocka, Trad.) México: Oxford University Press

Tena Millan, J. (1989). Organización de la Empresa: Teoría y Aplicaciones. Barcelona: Ediciones EADA Gestión.

Vigo, J. (1999). Manuales de Organización, Funciones y Lineamientos Organizacionales. (Material del Curso Anual de Especialización Organización, Métodos y Sistemas). Lima: Pontificia Universidad Católica del Perú.

Segura, S. (1978). Técnica y Práctica del Diseño Organizacional. Lima: Comunidad Industrial de SIDERPERU. 